\title{
Uncertainty analysis for flood inundation modelling with a random floodplain roughness field
}

Ying Huang ${ }^{1}$ and Xiaosheng Qin ${ }^{2^{*}}$

\begin{abstract}
Background: This study tested a first-order perturbation method based on Karhunen-Loevè expansion (FP-KLE), to analyze flood inundation modeling under uncertainty. The floodplain roughness over a 2-dimensional domain was assumed to be a statistically heterogeneous field with log-normal distributions. Firstly, we attempted to use KLE to decompose the random field of log-transferred floodplain roughness $N(x)$, which was based on the eigenvalues and eigenfunctions of the covariance function of $N(\boldsymbol{x})$, and a set of orthogonal normal random variables. Secondly, the maximum flow depths were expanded by the first-order perturbation method by using the same set of random variables as used in the KLE decomposition. Then, a flood inundation model, named FLO-2D, was adopted to numerically solve the corresponding perturbation expansions.

Results: To illustrate the methodology, a one-in-five-years flood event was chosen as the study case. The results indicated that the mean of the maximum flow-depth field obtained from the proposed method was fairly close to that from Monte Carlo Simulation (MCS), but the standard deviation was somewhat higher. However, the FP-KLE method was computationally more efficient than MCS.
\end{abstract}

Conclusions: The study verified the applicability of FP-KLE in handling uncertainties of flood modeling in a more efficient manner; further test with multiple inputs of random fields is desired.

Keywords: Karhunen-Loevè expansion; Roughness coefficient; Flood inundation modelling; Monte Carlo simulations

\section{Background}

Flood inundation mapping is important for helping identify potential flood profiles, and assist decision makers in planning and mitigation operations (Jung and Merwade, 2012). Due to inherent complexity and large numbers of parameters, flood inundation modelling processes often involve large uncertainties, especially at topographical formation where no sufficient data are available for model parameterization, calibration and validation. As a key global parameter in flood inundation modelling, it has been recognized that the Manning's roughness coefficient (Manning's $n$ ) could notably affect flood inundation predictions (Beven and Binley, 1992; Aronica et al., 1998;

\footnotetext{
* Correspondence: xsqin@ntu.edu.sg

${ }^{2} \mathrm{~S}$ chool of Civil \& Environmental Engineering and Institute of Catastrophe Risk Management (ICRM), Nanyang Technological University, Singapore 639798, Singapore

Full list of author information is available at the end of the article
}

Hall et al., 2005). Furthermore, due to different types of vegetation, surface irregularity and non-uniform/unsteady flows over the floodplain, the roughness coefficient has demonstrated a high heterogeneity (Pappenberger et al., 2005; Liu, 2009). Previously, many studies were devoted to analyse uncertainty propagation from manning's roughness coefficients during flood modelling, such as Generalized Likelihood Uncertainty Estimation (GLUE) and traditional Monte Carlo simulations (MCS) (Aronica et al., 1998; Aronica et al., 2002; Van Vuren et al., 2005; Reza Ghanbarpour et al., 2011; Jung and Merwade, 2012). However, most of these studies tend to adopt homogenous values of roughness coefficient over the study domain, and this could lead to significant discrepancies between the observed data and simulation results.

It has been realized that the geological-related process involving spatial distribution of uncertainty, such as roughness coefficients, can be more practically considered as a 
random field (Ghanem and Spanos, 1991; Lu and Zhang, 2007). Subsequently, the governing equations describing one- or multi-dimensional physical processes will be transformed into stochastic. The simulation results (such as the flow depth and flow velocity) are probabilistic distributions of the outputs, instead of deterministic quantifications. In recent decades, new stochastic methodologies based on Karhunen-Loevè expansion (KLE) decomposition have been proposed, which could consider the spatial distribution of uncertain parameters in generating realizations (Huang et al., 2001; Zhang and Lu, 2004; Liu et al., 2006). KLE decomposition was firstly developed to deal with uncertain random field with spatial distribution and the corresponding uncertainties were treated with an orthogonal basis of polynomial chaos (Ghanem and Spanos, 1991). Roy and Grilli (1997) firstly applied KLE to decompose the random field of hydraulic conductivity in a 2-dimensional (2D) stationary groundwater flow modelling system. Later on, Zhang and $\mathrm{Lu}$ (2004) developed a new approach called KLME (which combined KLE with moment equation method) and applied it to analyze the first and second moments of the hydraulic head. Despite of quite a large number of analytical approaches been developed for assessing parameter uncertainty in flood modeling, studies on the uncertainty parameters with spatial distribution are relatively limited. Liu and Matthies (2010) attempted to combine KLE and Hermite polynomial chaos expansion and examine the uncertainty from inflow, topography and roughness coefficient over the entire flood modelling domain using stochastic 2D shallow water equations. In this study, KLE is to be tested in decomposing the random field of floodplain roughness coefficients (keeping the channel roughness coefficients fixed) within a coupled 1-dimensional (1D) (for channel flow) and 2D (for floodplain flow) physical flood inundation model (i.e. FLO-2D). The method is effective in saving computational efforts without compromising the accuracy of uncertainty assessment. Previously, no such attempt was made using FLO-2D.

\section{Results and discussion \\ Study case}

A flood inundation case modified from Aronica et al. (2002) is chosen to demonstrate the proposed FP-KLE method. The related settings are listed as follows (Aronica et al., 2002; Hall et al., 2005; Bates et al., 2008): (i) 50-meter resolution DEM is used as the topographical data with 0.25 -meter accuracy vertically, changing from 67.73 to $83.79 \mathrm{~m}$; this is relatively moderate compared to those of the steeply-changing mountain areas; and (ii) channel cross-section is defined as rectangular with the size of $25 \mathrm{~m}$ (width) by $1.5 \mathrm{~m}$ (depth); (iii) the upstream inflow hydrograph is suggested as steady at $73 \mathrm{~m}^{3} / \mathrm{s}$; this corresponds to a flood event with a 5 -years return period (i.e. December 1992 flood lasting about 27.8 hours). The channel roughness coefficient $n$ is fixed at 0.03 . More detailed description of the study case can be referred to Bates et al. (2008). For uncertainty assessment, the floodplain roughness is assumed as a random field with lognormal probability distribution function (PDF). Monte Carlo simulation (MCS), as a traditional stochastic method, could be used to deal with the stochastic partial differential equations, i.e., 2D shallow water equations. Although MCS is easy to use, it is restricted by extensive computational needs, particularly for large-scale flood inundation modelling problems. It is desired to apply KLE method for improving the computational efficiency.

\section{Simplifications of KLE for the random floodplain roughness field}

In order to make the uncertainty analysis with KLE more practical, some simplifications are made according to Zhang and $\mathrm{Lu}$ (2004) for 1D model: (i) $\hat{\boldsymbol{x}}=\boldsymbol{x} / L_{x}$, where $L_{x}$ is the length of domain at the $\mathrm{x}$ direction, (ii) $\hat{\lambda}_{i}=\lambda_{i} / L_{x}$, (iii) normalized correlation length $\hat{\eta}=\eta / L_{x}$, and (iv) normalized eigenfuctions $\hat{f}_{i}(\boldsymbol{x})=f_{i}(\boldsymbol{x}) \sqrt{L_{x}}$. For simplicity, the tilde is omitted for the above four expressions in the followed equations and expressions within the parts of results and discussion, and conclusion.

The log floodplain roughness coefficient is assumed an exponential spatial covariance function (Roy and Grilli, 1997):

$$
C_{N}(\boldsymbol{x}, \boldsymbol{y})=C_{N}\left(x_{1}, x_{2}, y_{1}, y_{2}\right)=\sigma_{N} e^{\left[-\frac{\left|x_{1}-y_{1}\right|}{\eta_{x}}-\frac{\left|x_{2}-y_{2}\right|}{\eta_{y}}\right]}
$$

where $\eta_{x}$ and $\eta_{y}$ represent normalized correlation lengths in the $\mathrm{x}$ - and $\mathrm{y}$-directions, respectively; $\left(x_{1}, y_{1}\right)$ and $\left(x_{1}, y_{1}\right)$ are the spatial Cartesian coordinates of two points located in a $2 \mathrm{D}$ physical domain.

For a 2D rectangular modelling domain, we have eigenvalues for a $2 \mathrm{D}$ field and can integrate them as (Zhang and $\mathrm{Lu}, 2004$ ):

$$
\sum_{n=1}^{\infty} \lambda_{n}=\left(\sum_{i=1}^{\infty} \lambda_{i} \sum_{j=1}^{\infty} \lambda_{j}\right) / \sigma_{N}{ }^{2}=D \sigma_{N}{ }^{2}
$$

where $\lambda_{n}$ are the eigenvalues for 2D modelling domain; $\lambda_{i}(i=1,2, \ldots, \infty)$ and $\lambda_{j}(j=1,2, \ldots, \infty)$ are the eigenvalues for the $\mathrm{x}$ - and $\mathrm{y}$-directions separately; $\sigma_{N}$ is the standard deviation of the log-transformed floodplain roughness coefficient; $D$ is the size of the 2D modelling domain.

In this study, the random field of floodplain roughness coefficient $n(\boldsymbol{x})$ is suggested as log-normal PDF with $u_{n}=$ 0.06 (mean) and $\sigma_{n}=0.001$ (standard deviation), spatially. The $N(\boldsymbol{x})=\ln [n(\boldsymbol{x})]$ is a normal distribution with mean $u_{N}=-2.936$ and standard deviation $\sigma_{N}=0.495$. The range of $N(\boldsymbol{x})$ is approximated as $\left[u_{N}-3 \sigma_{N}, u_{N}+3 \sigma_{N}\right]$ which is $(0.012,0.234)$. To achieve both efficiency in 
operationality and accuracy in computation, the number of KLE terms with different normalized correlation lengths may vary with different scenarios (i.e., various scales of the domain size) with specific model settings (i.e., boundary condition settings) and floodplain roughness coefficients (i.e., changing from rural to urban areas) under consideration. In this case, the numbers of terms retained in KLE expansion in the $\mathrm{x}$-direction $\left(m_{x}\right)$ and y-direction $\left(m_{y}\right)$ are set as 20 and 10, respectively; hence, the total number of KLE terms is $20 \times 10=200$.

The eigenvalues would monotonically reduce as index $n$ increases, as shown in Equation (2). Figure 1(a) shows that, for different exponential spatial covariance functions (with different normalized correlation length $\eta$ ), the declining rate $\bar{\lambda}_{n}\left(\bar{\lambda}_{n}=\lambda_{n} /\left(D \sigma_{N}^{2}\right)\right)$ is different. When $\eta$ becomes larger, $\bar{\lambda}_{n}$ would reduce more significantly. Figure 1(b) illustrates that the summation of $\bar{\lambda}_{n}$ based on a finite number of terms rather than on an infinite number, can be considered as a function of the index $n$. The value of $\sum \bar{\lambda}_{n}$ would gradually approach to 1 when $n$ is increasing.

For this study case, the normalized correlation lengths are set as $\eta_{x}=0.15$ and $\eta_{y}=0.3$, and the total number of KLE terms is $m=200$. Figure 2 shows the decreasing rate of eigenvalues and how much energy of KLE approximation is obtained. For example, if $200 \mathrm{KLE}$ terms of $N(\boldsymbol{x})$ expansion are used in KLE decomposition and the total energy of the approximation would save by $86.56 \%$, as shown in Figure 2(b). Figures 3 shows two representations of the random fields of floodplain roughness coefficients over the 2D flood modelling domain with $\eta_{x}=0.15$ and $\eta_{y}=0.3$ and the number of KLE terms $=200$. These figures show that the KLE decomposition of the uncertain random field is different from the Monte Carlo sampling, in which the heterogeneous profile of random field can be represented by smoother eigenpairs as expressed in Equation (2).

\section{Comparison with MCS}

In order to verify the accuracy of the FP-KLE, the modelling results from 5,000 realizations of Monte Carlo simulations are also presented. Figure 4 shows the distribution statistics of the maximum flow depths $h(\boldsymbol{x})$ using KLE and MCS, respectively. From Figures 4a) and 4(b), it can be seen that the mean of $h(\boldsymbol{x})$ distribution (varying from 0 to $2.5 \mathrm{~m}$ ) simulated by KLE reproduces well the result from MCS. From Figures 4(c) and 4(d), different distributions of the standard deviation of $h(\boldsymbol{x})$ are found. The standard deviation of $h(x)$ simulated by FPKLE is somewhat higher than that calculated by MCS. This may because FP-KLE is in lower order (i.e. firstorder) and less capable of achieving a high accuracy, comparing with MCS.

Figure 5 shows a comparison of the statistics of the $h$ $(\boldsymbol{x})$ field along the cross-section $x=43$ between FP-KLE and MCS. It seems that the mean of the $h(x)$ along the concerned cross section simulated by FP-KLE fits very well with that simulated by MCS. However, the standard deviation from the perturbation method is higher than that from MCS. For example, at the location $(x, y)=(43$, 30 ), the standard deviation of the $h(x)$ (i.e. $0.01262 \mathrm{~m}$ ) is about $66.42 \%$ higher than that (i.e. $0.007583 \mathrm{~m}$ ) calculated by MCS; the range of the standard deviation by FP-KLE is from 0 to $0.01262 \mathrm{~m}$, and that by MCS is from 0 to $0.00883 \mathrm{~m}$. It indicates that the FP-KLE with 200 terms may not sufficiently capture the simulated standard deviation results by MCS.

Generally, the FP-KLE is capable of quantifying uncertainty propagation in a highly heterogeneous flood modelling system. By comparison, FP-KLE is proved to be more efficient than traditional MCS in terms of computational (a)

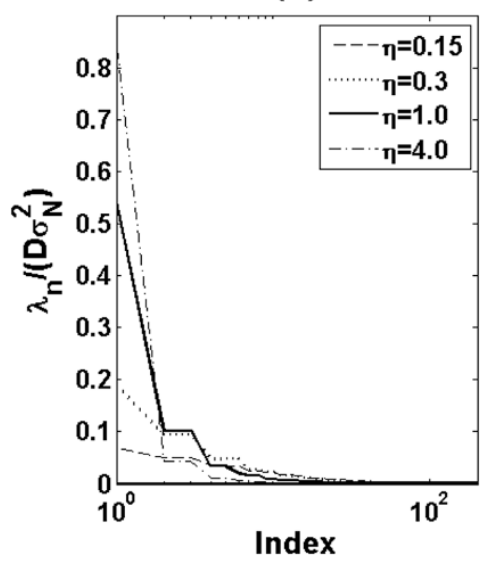

(b)

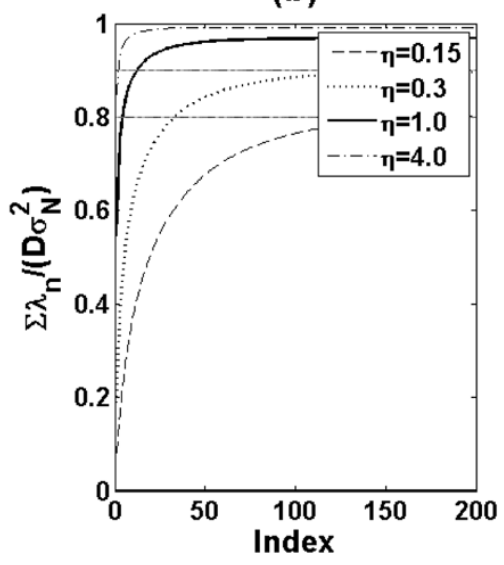

Figure 1 (a) Series of $\bar{\lambda}_{n}\left(\bar{\lambda}_{n}=\lambda_{n} /\left(D \sigma_{N}^{2}\right)\right)$ and (b) their finite cumulative sums for the 2D rectangular domain with different exponential spatial covariance functions (i.e. $\eta=\eta_{x}=\eta_{y}=0.15,0.3,1.0$ and 4.0 respectively). 

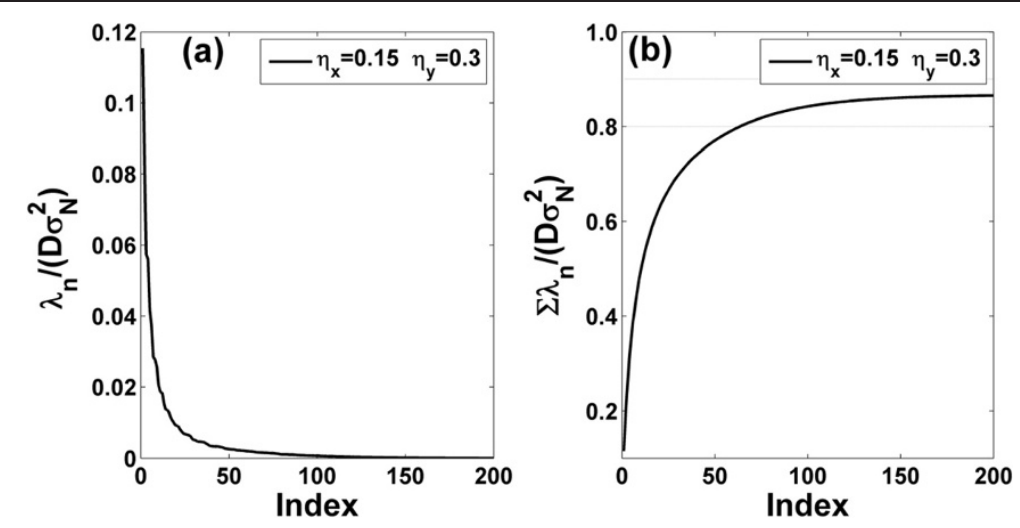

Figure 2 (a) Series of $\bar{\lambda}_{n}$, and (b) its finite cumulative sum for 2D rectangular domain with defined exponential spatial covariance function.

efforts. The presented approach can be used for largescale flood domains with high spatial-variability of input parameters, and it could provide reliable predictions to the decision-makers in flood risk assessment with relatively a small number of model runs.

\section{Conclusions}

This study attempted to use a first-order perturbation called FP-KLE to investigate the impact of uncertainty associated with floodplain roughness coefficients on a $2 \mathrm{D}$ flooding modelling process. Firstly, the KLE decomposition

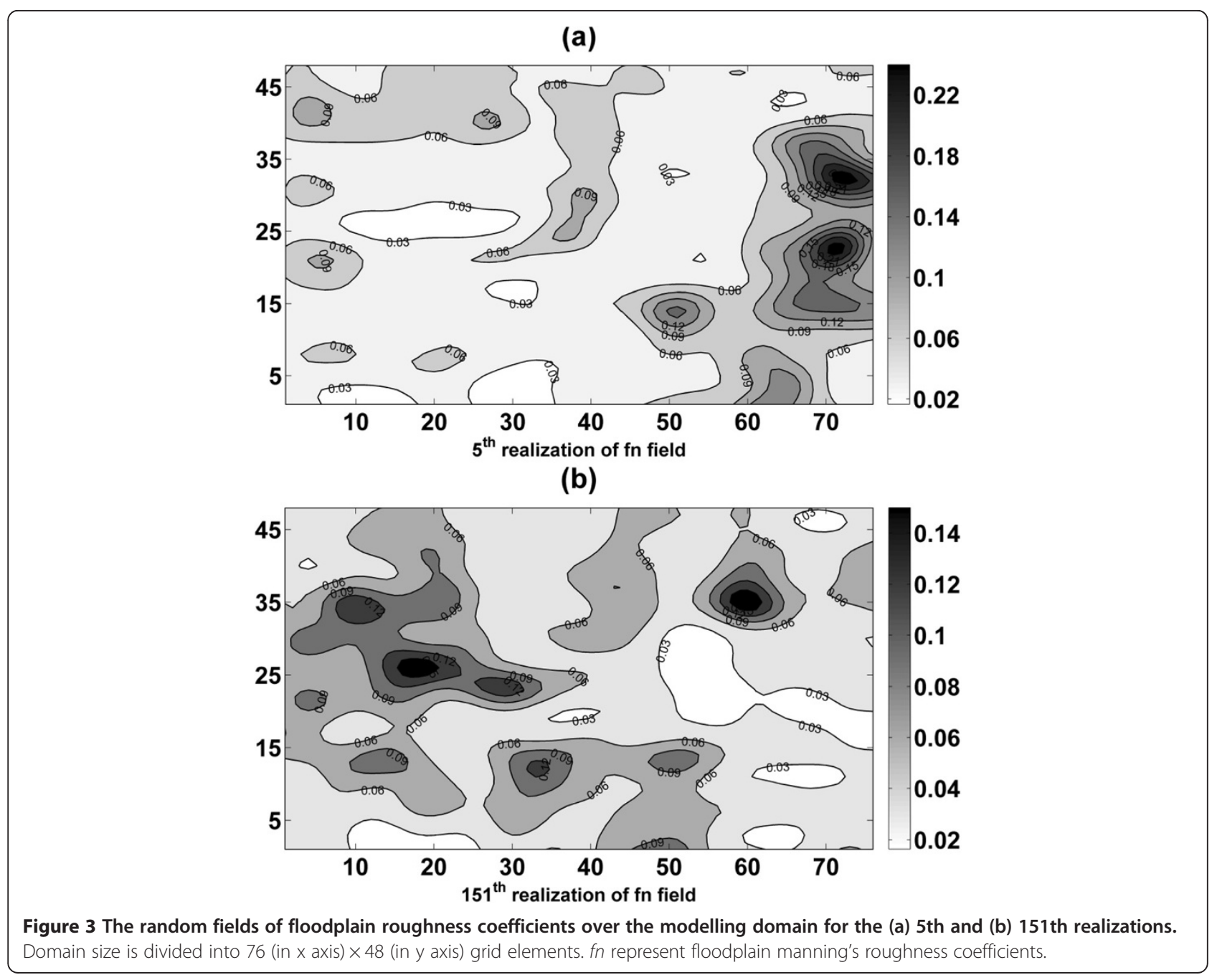



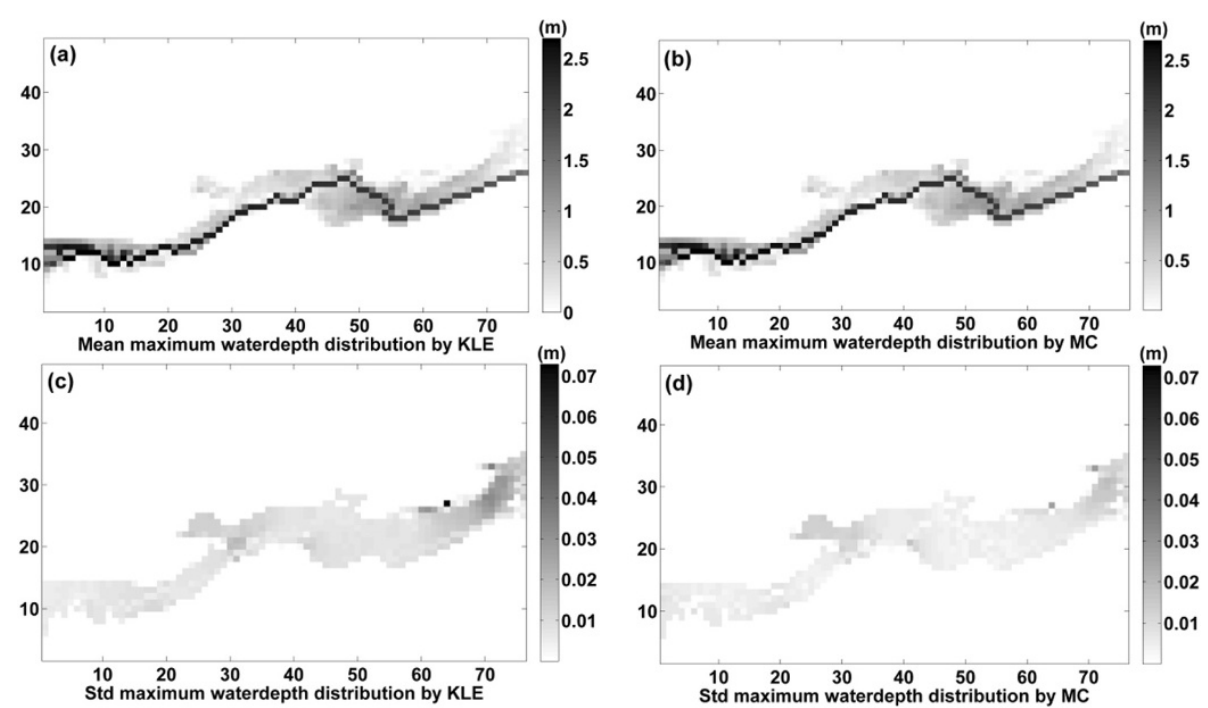

Figure 4 Comparison of statistics of maximum flow depth field simulated by the first-order perturbation based on the KLE and MCS: (a) and (b) are the mean maximum depth distributions calculated by FP-KLE and MCS, respectively; (c) and (d) are the standard deviation of the maximum depth distributions calculated by FP-KLE and MCS, respectively. Domain size is divided into 76 (in $\times$ axis) $\times 48$ (in y axis) grid elements.

for the log-transformed floodplain random field was made within a $2 \mathrm{D}$ rectangular flood domain represented by pairs of eigenvalue and eigenfunctions. Secondly, the first-order expansion of $h(\boldsymbol{x})$ perturbation was applied to the maximum flow depth distribution. Thirdly, the flood inundation model, i.e. FLO-2D, was used to solve each term of the perturbation based on the FP-KLE approach. Finally, the results were compared with those obtained from traditional Monte Carlo simulation (MCS).

The following facts were found from this study: (i) for the $2 \mathrm{D}$ flood case, with parameter setting of $\eta_{x}=0.15, \eta_{y}=$ 0.3 , and the number of KLE terms $=200$, about $86.56 \%$ energy have been saved; this was considered sufficient for reproduction of statistical characteristics; (ii) the mean of $h$ (x) field from FP-KLE reproduced well the results from MCS, but the standard deviation was somewhat higher; (iii) the first-order KLE-based perturbation method was computationally more efficient than MCS with comparable accuracy. Some limitations need further discussions in future studies: (i) compared with the first-order KLE-based perturbation approach, the second-order (or higher orders) perturbation may lead to more accurate result, but the required computational effort would increase dramatically; further test of the method on higher orders is desired; (ii) for this study, the simulation is in a steady-state condition; the KLE-based perturbation method for unsteady state could be further explored; (iii) the input random field in this study was assumed in normal distribution, non-normal
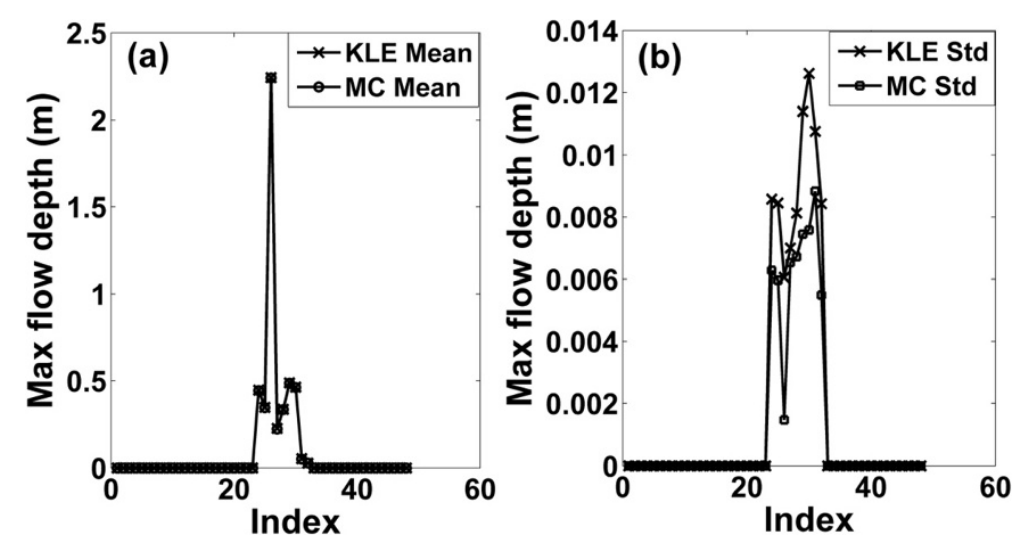

Figure 5 Comparison of statistics of max flow depth field simulated by FP-KLE and MCS along the profile $x=43 / 76$ : (a) mean max flow depth, and (b) standard deviation of max flow depth. 
distributions of the input random fields could be explored in the future.

\section{Methods}

\section{Stochastic flood inundation model}

FLO-2D is used in this study as the flood inundation model. It is based on the full 2D shallow water equations (also called dynamic wave momentum equation) (FLO2D Software, 2012). In the FLO-2D modeling system, channel flow is 1D with the channel geometry represented by either rectangular or trapezoidal cross sections; meanwhile, the overland flow is modelled $2 \mathrm{D}$ as either sheet flow or flow in multiple channels (FLO-2D Software, 2012). Overbank flow in the channel is computed when the channel capacity is exceeded. Besides, an interface routine calculates the channel to floodplain flow exchange including return flow to the channel. More technical details of FLO-2D can be referred to Obrien et al. (1993), O'Brien et al. (1999), and D'Agostino and Tecca (2006).

To describe a 2D flood inundation process, shallow water equations can be used (Chow et al., 1988; Obrien et al., 1993; FLO-2D Software, 2012):

$$
\begin{aligned}
& \frac{\partial h(\boldsymbol{x})}{\partial t}+\nabla[h(\boldsymbol{x}) V]=I \\
& S_{f}=S_{o}-\nabla h(\boldsymbol{x})-\frac{1}{g}(V \cdot \nabla) V-\frac{1}{g} \frac{\partial V}{\partial t} \\
& S_{f}=\frac{n(\boldsymbol{x})^{2}}{R^{4}} V|V|
\end{aligned}
$$

where, $h(\boldsymbol{x})$ is the flow depth; $V$ represents the averagedin-depth velocity in each direction $\boldsymbol{x} ; \boldsymbol{x}$ represents Cartesian coordinate spatially, such as $x=(x, y)$ represents 2D Cartesian coordinate; $t$ is the time; $S_{o}$ is the bed slope, and $S_{f}$ is the friction slope; and $I$ is lateral flow into the channel from other sources. Equation (3a) is the continuity equation or mass conservation equation, and Equation (3b) is the momentum equation; both of them are the fundamental equations in the flood modelling. In Equation (3c), $n$ is the Manning coefficient (Manning's $n$ ), which is the most commonly applied friction parameter in flooding modelling. $R$ is the hydraulic radius. Equations (3a)-(3c) are solved mathematically in eight directions by FLO-2D. In this study, $n(\boldsymbol{x})$ is assumed as a random function spatially, and Equations (3a)-(3c) are transformed into stochastic partial differential equations with random floodplain roughness coefficients and other items within the model are considered to be deterministic. Our purpose is to solve the mean and standard deviation of the flow depth $h(x)$, which are used to assess the uncertainty propagation during the flood inundation modelling.

\section{Karhunen-Loevè expansion (KLE) of log floodplain manning's roughness coefficients}

Let the $n(\boldsymbol{x})$ be a random field of floodplain manning's roughness coefficient, and $N(\boldsymbol{x})=\ln [n(\boldsymbol{x}, \omega)]$, where $\boldsymbol{x} \in$ $D$ and $\omega \in \Omega$ (a probabilistic space). This means $N(x)$ changes spatially. Its covariance function $C_{N}(\boldsymbol{x}, \boldsymbol{y})=\left\langle N^{\prime}(\boldsymbol{x}, \omega)\right.$ $N^{\prime}(\boldsymbol{y}, \omega)>$ is assumed as bounded, symmetric, and positive, which can be expressed as (Ghanem and Spanos, 1991):

$$
C_{N}(\mathbf{x}, \boldsymbol{y})=\sum_{n=1}^{\infty} \lambda_{n} f_{n}(\boldsymbol{x}) f_{n}(\boldsymbol{y})
$$

where $\lambda_{n}$ represent eigenvalues; $f_{n}(\boldsymbol{x})$ are eigenfunctions, which are orthogonal and determined by dealing with the Fredholm equation analytically or numerically as (Courant and Hilbert, 1989):

$$
\int_{D} C_{N}(\mathbf{x}, \boldsymbol{y}) f_{n}(\boldsymbol{x}) d \boldsymbol{x}=\lambda_{n} f_{n}(\boldsymbol{y})
$$

where $\lambda_{n}$ and $f_{n}(\boldsymbol{x})$ for some specific covariance functions could be solved analytically (Zhang and Lu, 2004). The KLE representation of floodplain roughness coefficients can be expressed as:

$$
N(\boldsymbol{x}, \omega)=\mu(\boldsymbol{x})+\sum_{n=1}^{\infty} \xi_{n}(\omega) \sqrt{\lambda_{n}} f_{n}(\boldsymbol{x})
$$

where $\mu(\boldsymbol{x})$ represents the mean of $N(\boldsymbol{x}, \omega)$; and $\xi_{n}(\omega)$ are the orthogonal random variables with standard Gaussian distribution. For conciseness, $\omega$ is omitted in following related expressions and equations. The calculated $\lambda_{n}$ can be ranked as monotonically decreasing series with index $n$. In order to make the approximation effective in both computational effort and accuracy, the KLE representation of $N(\boldsymbol{x})$ can be written in a finite form as follows (Liu et al., 2006; Li and Zhang, 2007):

$$
\tilde{N}(\boldsymbol{x})=\mu(\boldsymbol{x})+\sum_{n=1}^{m} \xi_{n} \sqrt{\lambda_{n}} f_{n}(\boldsymbol{x})
$$

Since $\sqrt{\lambda_{n}}$ and $f_{n}(\boldsymbol{x})$ always appear in pairs, let $g_{n}(\boldsymbol{x})=\sqrt{\lambda_{n}} f_{n}(\boldsymbol{x})$. Then, Equation (7) can be simplified into:

$$
\tilde{N}(\boldsymbol{x})=\mu(\boldsymbol{x})+\sum_{n=1}^{m} \xi_{n} g_{n}(\boldsymbol{x})
$$

where $\tilde{N}(\boldsymbol{x})$ is the approximation for the random floodplain roughness field. Generally, the more the terms used in $g_{n}(\boldsymbol{x})$, the more accurate the random floodplain roughness filed is represented; this implies that more random field energy is saved, but with an increasing computational efforts. For simplicity of expression, the tilde over the symbol $N$ is omitted in the followed expressions and functions. 


\section{Perturbation method}

In this study, the fluctuation of the max flow depths, as one of the important indicators of the flood inundation simulation, is affected by the spatial variability of the floodplain roughness values $N(\boldsymbol{x})$. The maximum flow depths $h(x)$ can be expressed with a perturbation expansion in an infinite series as follows (Phoon et al., 2002; Liu et al., 2006; Li and Zhang, 2007; Lu and Zhang, 2007):

$$
h(\boldsymbol{x})=\sum_{i=0}^{\infty} h^{(i)}(\boldsymbol{x})
$$

where, $h^{(i)}(\cdot)$ is the $i^{\text {th }}$ order perturbation term based on the standard deviation of $N(\boldsymbol{x})$ (denoted as $\sigma_{N}$ ).

Substituting Equations (8) and (9) into Equations $(3 \mathrm{a}-3 \mathrm{c})$, we can obtain the $i^{\text {th }}$ order term of the expansion $h^{(i)}(\boldsymbol{x})$ and each order of perturbation is calculated based on $\sigma_{N}$. For example, the first-order perturbation expansion for $h(\boldsymbol{x})$ can be expressed as $h(\boldsymbol{x})=h^{(0)}(\boldsymbol{x})+h^{(1)}(\boldsymbol{x})$. It can be seen that the higher the order of the term $h^{(i)}(\cdot)$ kept in the expansion of $h(\boldsymbol{x})$, the more energy could be retained in the expansion; hence more corrections are provided for the statistical moments (i.e. mean and variation) of the simulation results (Roy and Grilli, 1997). However, in this study, considering the computational requirements of the flood modelling, only the first-order perturbation expansion based on KLE is investigated.

\section{Competing interests}

The authors declare that they have no competing interests.

\section{Authors' contributions}

YH carried out the modelling studies and drafted the manuscript; XSQ helped out in the result analysis, and participated in the coordination and refinement of the manuscript. Both authors read and approved the final manuscript.

\section{Acknowledgement}

This research was supported by Singapore's Ministry of Education (MOM) AcRF Tier 1 Project (M4010973.030) and Tier 2 Project (M4020182.030).

\section{Author details}

${ }^{1}$ School of Civil \& Environmental Engineering, Nanyang Technological University, Singapore 639798, Singapore. ${ }^{2}$ School of Civil \& Environmental Engineering and Institute of Catastrophe Risk Management (ICRM), Nanyang Technological University, Singapore 639798, Singapore.

Received: 23 December 2013 Accepted: 10 February 2014 Published: 18 February 2014

\section{References}

Aronica G, Hankin B, Beven K (1998) Uncertainty and equifinality in calibrating distributed roughness coefficients in a flood propagation model with limited data. Adv Water Resour 22(4):349-365

Aronica G, Bates PD, Horritt MS (2002) Assessing the uncertainty in distributed model predictions using observed binary pattern information within GLUE. Hydrol Process 16(10):2001-2016

Bates P, Fewtrel T, Trigg M, Neal J (2008) LISFLOOD-FP User manual and technical note, code release 4.3.6. University of Bristol

Beven K, Binley A (1992) The future of distributed models: model calibration and uncertainty prediction. Hydrol Process 6(3):279-298

Chow VT, Maidment DR, Mays LW (1988) Applied hydrology. McGraw-Hill International Editions, New York
Courant R, Hilbert D (1989) Methods of Mathematical Physics. Interscience Publishers, New York

D'Agostino V, Tecca PR (2006) Some considerations on the application of the FLO-2D model for debris flow hazard assessment. In: Lorenzini G, Brebbia CA Emmanouloudis DE (eds) Monitoring, Simulation, Prevention and Remediation of Dense and Debris Flows. WIT Transactions on Ecology and the Envionment, 90, pp 159-170

FLO-2D Software, Inc (2012) FLO-2D Reference Manual 2009. https://www.flo-2d. com/download

Ghanem RG, Spanos PD (1991) Stochastic Finite Elements: A Spectral Approach Springer, New York

Hall J, Tarantola S, Bates PD, Horritt MS (2005) Distributed sensitivity analysis of flood inundation model calibration. Journal of Hydraulic Engineering-ASCE 131(2):117-126

Huang SP, Quek ST, Phoon KK (2001) Convergence study of the truncated Karhunen-Loeve expansion for simulation of stochastic processes. Int J Numer Methods Eng 52(9):1029-1043

Jung Y, Merwade V (2012) Uncertainty Quantification in Flood Inundation Mapping Using Generalized Likelihood Uncertainty Estimate and Sensitivity Analysis. J Hydrol Eng 17(4):507-520

Li H, Zhang D (2007) Probabilistic collocation method for flow in porous media: Comparisons with other stochastic methods. Water Resour Res 43(9):W09409, doi:10.1029/2006WR005673

Liu D (2009) Uncertainty Quantification with Shallow Water Equations. Thesis, Institute of Scientific Computing, Technical University of Braunschweig, Germany

Liu DS, Matthies HG (2010) Uncertainty quantification with spectral approximations of a flood model. 9th World Congress on Computational Mechanics and 4th Asian Pacific Congress on Computational Mechanics, WCCM/APCOM 2010, July 19, 2010 - July 23, 2010. Institute of Physics Publishing, Sydney, NSW, Australia

Liu G, Zhang D, Lu Z (2006) Stochastic uncertainty analysis for unconfined flow systems. Water Resour Res 42(9), W09412

Lu Z, Zhang D (2007) Stochastic Simulations for Flow in Nonstationary Randomly Heterogeneous Porous Media Using a KL-Based Moment-Equation Approach. Multiscale Modeling \& Simulation 6(1):228-245

O'Brien JS, Julien PY, Fullerton WT (1993) Two-dimensional water flood and mudflow simulation. J of Hydraulic Engineering-ASCE 119(2):244-261

O'Brien JS, Fullerton WT, Finch DM, Whitney JC, Kelly JF, Loftin SR (1999) Simulation of Rio Grande floodplain inundation Using FLO-2D. In: Rio Grande ecosystems: linking land, water, and people: toward a sustainable future for the Middle Rio Grande Basin. 1998 June 2-5. U.S. Department of Agriculture, Forest Service, Rocky Mountain Research Station, Albuquerque, NM. Proc. RMRS-P-7. Ogden, UT, pp 52-60

Pappenberger F, Beven K, Horritt M, Blazkova S (2005) Uncertainty in the calibration of effective roughness parameters in HEC-RAS using inundation and downstream level observations. J Hydrol 302(1-4):46-69

Phoon KK, Huang SP, Quek ST (2002) Simulation of second-order processes using Karhunen-Loeve expansion. Comput Struct 80(12):1049-1060

Reza Ghanbarpour M, Salimi S, Saravi MM, Zarei M (2011) Calibration of river hydraulic model combined with GIS analysis using ground-based observation data. Res J Appl Sci Eng Technol 3(5):456-463

Roy RV, Grilli ST (1997) Probabilistic analysis of flow in random porous media by stochastic boundary elements. Engineering Analysis with Boundary Elements 19(3):239-255

Van Vuren S, De Vriend H, Ouwerkerk S, Kok M (2005) Stochastic modelling of the impact of flood protection measures along the river waal in the Netherlands. Nat Hazards 36(1-2):81-102

Zhang D, Lu Z (2004) An efficient, high-order perturbation approach for flow in random porous media via Karhunen-Loève and polynomial expansions. J Comput Phys 194(2):773-794

\section{doi:10.1186/2193-2697-3-9}

Cite this article as: Huang and Qin: Uncertainty analysis for flood inundation modelling with a random floodplain roughness field. Environmental Systems Research 2014 3:9. 